\title{
Aplicación y evaluación de metodologías activas de enseñanza-aprendizaje que faciliten la adquisición de competencias relacionadas con los Sistemas de Información Geográfica en los programas de Grado y Máster de la Universidad de La Rioja
}

\author{
María Paz Diago Santamaría, Marisol Andrades Rodrígueza , Jesús María Aransay Azofra ${ }^{b}$, José \\ Ángel Llorente Adán ${ }^{c}$, Purificación Ruiz Flaño ${ }^{c}$ y Noemí Solange Lana-Renault Monrealc
}

a Departamento de Agricultura y Alimentación (Universidad de La Rioja. C/ Madre de Dios 53, 26006. España.),

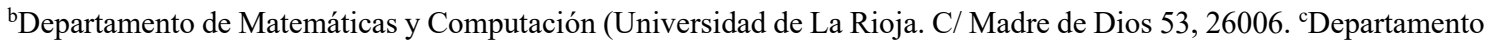
de Ciencias Humanas (Universidad de La Rioja. C/ Luis de Ulloa, 2, 26002 España.), España. mariapaz.diago@unirioja.es

\section{Abstract}

In recent decades, the use of information systems has become widespread, thanks to greater data availability, as well as the improvement of the power and capacity of computer systems and programs. Specifically, the management of geographical data through the so-called Geographic Information Systems (GIS) has brought about a revolution in the ability to obtain information and advanced science. In this work, a list of the Undergraduate and Master's subjects of the University of La Rioja in which active GIS teaching methodologies are applied has been identified. A self-evaluation survey has been designed and carried out that has allowed us to assess the starting point of the knowledge about GIS that students have. Likewise, in these subjects a novel didactic material created for GIS learning is being used and its structure responds precisely to the level of use and demand of GIS and geographic data in general and in particular of university students. With the results obtained, the efficiency of the methodology designed for learning can be analyzed.

Keywords: learning, methodology, geographic information systems, didactic material, survey evaluation

\section{Resumen}

En las últimas décadas se ha generalizado la utilización de sistemas de información, gracias a una mayor disponibilidad de datos, así como a la mejora de la potencia y capacidad de los sistemas y programas informáticos. En concreto, el manejo de los datos geográficos mediante los denominados Sistemas de Información Geográficos (SIG) ha supuesto una revolución en la capacidad de obtención de información y avance de la ciencia. En este trabajo se han identificado las asignaturas de Grado y Máster de la Universidad de la Rioja en las que se aplican metodologías activas de enseñanza de los SIG. Se ha diseñado y realizado una encuesta de autoevaluación que nos ha permitido valorar el punto de partida de los conocimientos sobre SIG de los que disponen los estudiantes. Así mismo, en estas asignaturas se está utilizando el material didáctico creado para el aprendizaje y cuya estructura responde precisamente al nivel de uso y demanda de los SIG y datos geográficos en general y en particular de los universitarios. Con los resultados obtenidos se puede analizar la eficiencia de la metodología diseñada para el aprendizaje

Palabras clave: aprendizaje, metodología, sistemas de información geográfica, material didáctico, encuesta 
Aplicación y evaluación de metodologías activas de enseñanza-aprendizaje que faciliten la adquisición de competencias relacionadas con los Sistemas de Información Geográfica en los programas de Grado y Máster de la Universidad de La Rioja

\section{Introducción}

El perfeccionamiento y uso de las tecnologías de la información y comunicación (TIC) se han convertido en una habilidad clave y necesaria para la obtención de información y el avance del desarrollo científico en todas las disciplinas del conocimiento. Así, en las últimas décadas se ha generalizado la utilización de sistemas de información, gracias a una mayor disponibilidad de datos, así como a la mejora de la potencia y capacidad de los sistemas y programas informáticos. Es el caso particular de los datos geográficos, cuyo manejo, mediante los denominados Sistemas de Información Geográficos (SIG) ha supuesto una revolución en la capacidad de obtención de información y avance de la ciencia.

Dentro del contexto universitario, los métodos de enseñanza-aprendizaje del actual Espacio Europeo de Educación Superior (EEES) han ido adaptándose progresivamente a la utilización y manejo de datos y de SIG, que ha sucedido en la práctica totalidad de las áreas de estudio de la universidad, también en la Universidad de La Rioja (Andrades et al., 2018, Llorente et al., 2019). La incorporación de las Tics y el uso de datos geográficos y SIG en el contexto universitario ha abordado, entre otros, los siguientes objetivos principales: 1. la formación en la utilización de herramientas y materiales que promuevan las tecnologías de la información de cara a conseguir las competencias profesionales para el futuro del alumnado (Cabero, 2005), y 2. fomentar las habilidades basadas en TIC para trabajar y vivir en la sociedad de la información actual (Guitero et al., 2008).

En la Universidad de La Rioja el proyecto de innovación docente "Coordinación y mejora de la docencia en asignaturas que utilizan Sistemas de Información Geográfica en la Universidad de La Rioja" realizó un diagnóstico de la utilización de los SIG en asignaturas de Grado y Máster impartidas. Dicho proyecto concluyó que los SIG se aplicaban en 19 asignaturas diferentes de 7 Grados y 4 Máster. Un paso más se dio en el siguiente proyecto "Coordinación y mejora en la utilización de SIG como una herramienta en la realización de TFGs, TFMs y Tesis Doctorales en la Universidad de La Rioja” en el que se abordó el análisis de la metodología de aplicación de los SIG en la elaboración de Trabajos Fin de Grado, Trabajos Fin de Máster y Tesis Doctorales en la Universidad de La Rioja desde su creación. Como resultado de este segundo proyecto se identificaron tres grupos de usuarios de SIG, en función del uso de datos geográficos y del software de tratamiento de información geográfica utilizado. Las actividades de los dos proyectos de innovación docente anteriormente mencionados se han materializado en una Publicación Docente titulada Enseñanza de Sistemas de Información Geográfica (SIG) en estudios de grado y posgrado en la Universidad de La Rioja. Principios teóricos y ejercicios prácticos (Andrades et al. 2020) que constituye una guía de referencia para docencia transversal sobre SIG y sus funcionalidades básicas y avanzadas (en relación a los tres grupos de usuarios descritos anteriormente) con guiones de prácticas para distintos niveles de uso de SIG, adaptables a las distintas asignaturas de Máster y Grado en las que se utilizan los SIG, en la Universidad de La Rioja.

\section{Objetivos}

Los objetivos de este trabajo son los siguientes:

1. Implementar la metodología y material didáctico sobre el uso y aplicaciones de los SIG en las distintas asignaturas de Grado y Máster de la Universidad de La Rioja.

2. Evaluar, mediante una ficha o rúbrica de autoevaluación, el nivel de conocimiento en el tratamiento de datos geográficos y de SIG de los estudiantes, con independencia de la disciplina. 
3. Mejorar los métodos de enseñanza-aprendizaje llevados a cabo en la Universidad de La Rioja desde una perspectiva multidisciplinar, y a partir de formas de actuación que conlleven innovación y coordinación entre profesores de distintas áreas de estudio. Este hecho aporta un valor añadido al trabajar de manera cooperativa distintas asignaturas, aunque con metodologías comunes. Un hecho que fomenta el intercambio de ideas, así como la reflexión crítica entre docentes involucrados en el progreso educativo universitario.

\section{Desarrollo de la innovación}

Para la consecución de los objetivos propuestos se ha seguido un sencillo esquema metodológico, que cuenta con los siguientes pasos:

1. Se han identificado las asignaturas de Grado y Máster de la Universidad de la Rioja en las que se aplican metodologías activas de enseñanza de los SIG. En total, se han identificado 18 asignaturas que forman parte del plan de estudios de seis Grados diferentes, así como en tres Masters (Tabla $1)$.

Tabla1. Asignaturas de los Grados y Másteres de la Universidad de La Rioja donde se utilizan metodologías de tratamiento de datos geográficos y de SIG.

\begin{tabular}{|c|c|c|}
\hline \multirow{15}{*}{ Grados } & \multirow{3}{*}{ Grado en Enología } & $\begin{array}{l}\text { Prácticas Integradas de } \\
\text { Viticultura }\end{array}$ \\
\hline & & Viticultura de precisión \\
\hline & & Geología, suelo y clima \\
\hline & \multirow{4}{*}{ Grado en Ingeniería Agrícola } & Geología, suelo y clima \\
\hline & & Informática \\
\hline & & $\begin{array}{l}\text { Prácticas Integradas de } \\
\text { Viticultura }\end{array}$ \\
\hline & & Informática \\
\hline & \multirow{4}{*}{ Grado en Geografía e Historia } & $\begin{array}{l}\text { Cartografía y representación } \\
\text { gráfica }\end{array}$ \\
\hline & & Geografía Física \\
\hline & & Patrimonio Natural \\
\hline & & Geografía y Medio Ambiente \\
\hline & \multirow{4}{*}{ Grado en Turismo } & Patrimonio Natural \\
\hline & & Geografía y Medio Ambiente \\
\hline & & Geografía del turismo y el ocio. \\
\hline & & $\begin{array}{l}\text { Planificación y gestión del } \\
\text { turismo en espacios naturales y } \\
\text { rurales. }\end{array}$ \\
\hline
\end{tabular}

(c) 2020, Universitat Politècnica de València

Congreso In-Red (2020) 
Aplicación y evaluación de metodologías activas de enseñanza-aprendizaje que faciliten la adquisición de competencias relacionadas con los Sistemas de Información Geográfica en los programas de Grado y Máster de la Universidad de La Rioja

\begin{tabular}{|c|c|c|}
\hline & Grado en Estudios Ingleses & Geografía y Medio Ambiente \\
\hline & $\begin{array}{l}\text { Grado en Lengua y Literatura } \\
\text { Hispánica }\end{array}$ & Geografía y Medio Ambiente \\
\hline \multirow{4}{*}{ Másteres } & $\begin{array}{l}\text { Master Universitario en Tecnold } \\
\text { Vitivinícola }\end{array}$ & Retos en Viticultura \\
\hline & $\begin{array}{l}\text { Máster en Ingeniería } \\
\text { Agronómica }\end{array}$ & $\begin{array}{l}\text { Ordenación y gestión del } \\
\text { territorio. }\end{array}$ \\
\hline & \multirow{2}{*}{$\begin{array}{l}\text { Máster en Estudios Avanzados } \\
\text { en Humanidades }\end{array}$} & $\begin{array}{l}\text { Nuevas técnicas aplicadas al } \\
\text { análisis del patrimonio } \\
\text { territorial. }\end{array}$ \\
\hline & & $\begin{array}{l}\text { Recursos naturales, paisaje y } \\
\text { evaluación del territorio }\end{array}$ \\
\hline
\end{tabular}

2. En estas asignaturas se utilizará el material didáctico recientemente publicado por el Servicio de Publicaciones de la Universidad: "Enseñanza de Sistemas de Información Geográfico (SIG) en estudios de Grado y Posgrado en la Universidad de La Rioja" con ISBN: 978-84-09-17400-3, dentro de la colección de Innovación Docente (Andrades et al. 2020). Este libro ha sido elaborado con la finalidad de facilitar la aplicación de los SIG mediante la ejecución de los ejercicios prácticos diseñados (Fig. 1).

Enseñanza de Sistemas de

Información Geográfica (SIG)

en estudios de grado

y posgrado en la

Universidad de La Rioja

Principios teóricos

y ejercicios prácticos

Marisol Andrades Rodriguez

Jesús Maria Aransay Azofra

M.'Paz Diago Santamaria

Noemi Solange Lana-Renault Monreal

José Ángel Llorente Adản

Purificadón Rub Flaño

Eduardo Sáenz de Cabezón Irigaray

\section{1.}

ATP UNEERSIDAD

Fig. 1. Portada del libro "Enseñanza de Sistemas de Información Geográfico (SIG) en estudios de Grado y Posgrado en la Universidad de La Rioja” publicado por la Universidad de La Rioja en 2020

(cc) EY-NC-ND 2020, Universitat Politècnica de València

Congreso In-Red (2020) 
De forma previa a la utilización de este material, se ha diseñado (con siete sencillas preguntas) y realizado una encuesta o cuestionario de autoevaluación entre los alumnos de los distintos Grados y Másteres, que nos ha permitido valorar el punto de partida de los conocimientos sobre SIG de los que éstos disponen. El cuestionario (Fig. 2) incluye indicadores de progreso medibles, que pueden ser confrontados con el cuestionario inicial.

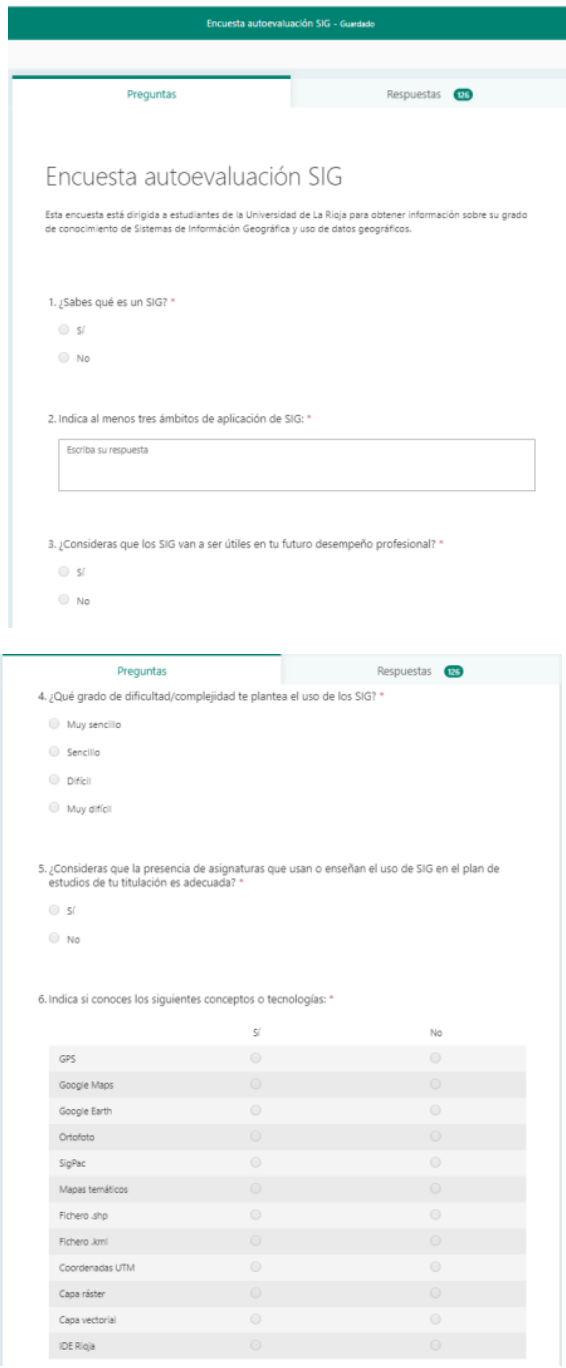

(a)

(b)

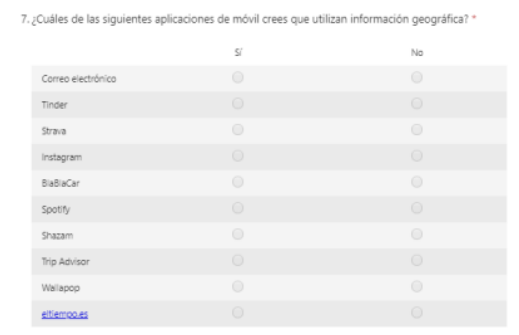

(c)

Figura 2. Encuesta de evaluación diseñada y realizada a los estudiantes de las asignaturas en que se utiliza el SIG en la Universidad de La Rioja. (a) Preguntas 1-3, (b) preguntas 4-6, y (c) pregunta 7.

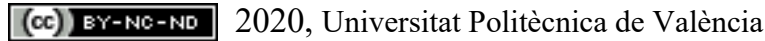

Congreso In-Red (2020) 
Aplicación y evaluación de metodologías activas de enseñanza-aprendizaje que faciliten la adquisición de competencias relacionadas con los Sistemas de Información Geográfica en los programas de Grado y Máster de la

Universidad de La Rioja

3. De forma paralela a la aplicación de este material didáctico se elaborará un cuestionario de autoevaluación o rúbrica que permita valorar la eficacia de la metodología aplicada. El cuestionario incluirá indicadores de progreso medibles, que pueden ser confrontados con el cuestionario inicial.

4. Con los resultados obtenidos tanto en la encuesta previa como en el cuestionario de autoevaluación se procederá a analizar la eficiencia de la metodología diseñada para el aprendizaje.

\section{Resultados}

Los resultados obtenidos al aplicar la metodología propuesta son los siguientes:

1. El cuestionario de evaluación ha sido realizado por los alumnos y los resultados obtenidos nos han permitido valorar el punto de partida de los conocimientos de los que disponen sobre SIG.

A la hora de interpretar los resultados hay que tener en cuenta algunos datos relevantes, entre los que cabe destacar por una parte que las encuestas han sido respondidas tanto por estudiantes de los primeros cursos de Grado como por los que están un Master y por otra, que los alumnos en cuestión pertenecen a titulaciones muy diversas, cuyo único elemento común es el uso de herramientas SIG en alguna asignatura de sus planes de estudios. El proceso de recogida de las respuestas se ha llevado a cabo mediante un formulario en Google Forms (Fig. 2) de forma totalmente anónima. Finalmente, consideramos también relevante indicar que el número de estudiantes que completaron el formulario fue de 126.

A continuación exponemos las preguntas realizadas y los resultados obtenidos en un gráfico circular con su leyenda correspondiente.

La $1^{a}$ pregunta que se formuló fue si tenían alguna noción previa de lo que era un SIG, en la que estudiando las respuestas se puede ver que un 50\% de ellos sí lo tenía (Fig. 3).

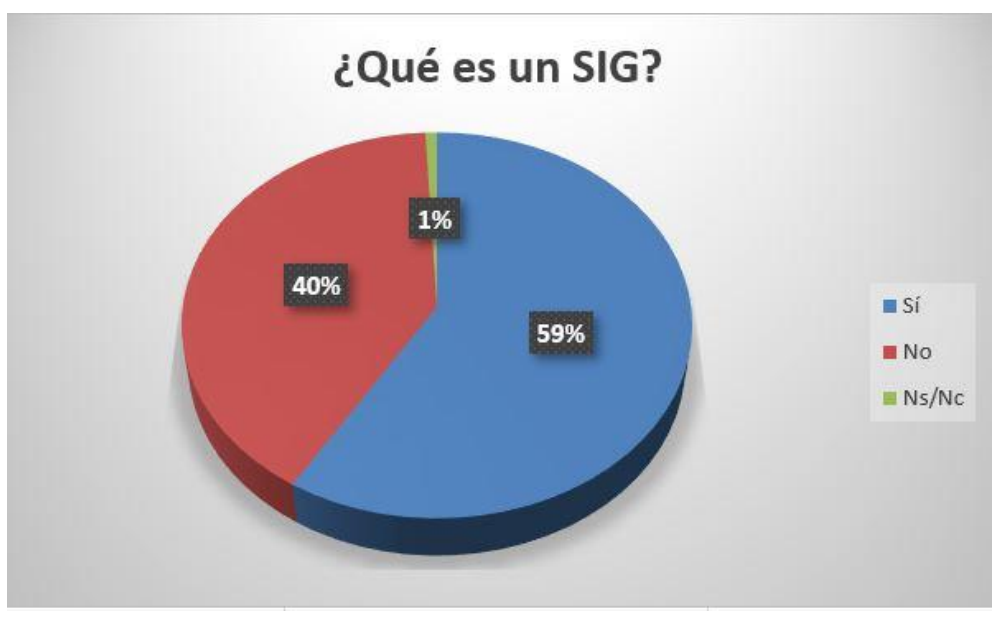

Fig. 3. Gráfico de resultados relativos al conocimiento de $S I G(n=126)$.

En la $2^{\mathrm{a}}$ pregunta se pedía que señalaran al menos tres ámbitos de aplicación de los SIG. Entre los estudiantes que habían respondido afirmativamente a la pregunta anterior aparece una gran dispersión entre los distintos usos identificados. Entre estos usos se mencionaba la medición e identificación de parcelas, la aplicación de fitosanitarios o la viticultura en general, usos en arqueología, en geografía social o incluso en cuestiones logísticas. 
En la tercera pregunta se les cuestionaba acerca de la percepción que tenían sobre la relevancia que los SIG podían tener en su futuro desempeño profesional. Una amplia mayoría de los estudiantes, a pesar de la dispersión de las titulaciones de las que procedían, consideraron que los SIG van a tener un papel relevante en su futuro laboral. También nos gustaría destacar que, incluso estudiantes que habían respondido negativamente a la primera pregunta, respondieron a esta pregunta de manera afirmativa (Fig. 4).

\section{¿Consideras que los SIG van a ser útiles en tu desempeño profesional?}

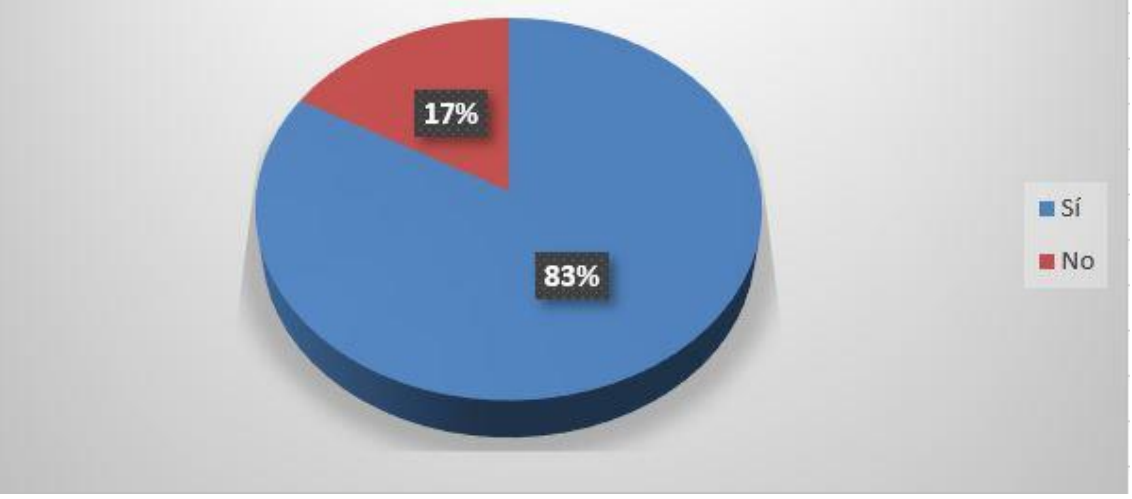

Fig. 4. Gráfico de resultados relativo a la relevancia de los $S I G$ en el desempeño profesional ( $n=126)$.

En la siguiente pregunta, la $4^{\mathrm{a}}$, se pretendía conocer la impresión subjetiva que los estudiantes tenían sobre la complejidad o dificultad de uso de los SIG. Mayoritariamente, los estudiantes percibían el aprendizaje de los SIG como difícil o muy difícil. Sería interesante conocer las razones por las que los estudiantes perciben esta dificultad, y nos parece que debe estar relacionada con el conocimiento previo que tengan algunos de ellos (el hecho de haberlo usado en algunas asignaturas cursadas previamente) así como la bastante habitual percepción de complejidad hacia las herramientas TIC que manifiestan los estudiantes de titulaciones "no técnicas" (Fig. 5).

\section{¿Qué grado de dificultad/complejidad te plantea el uso de los SIG?}

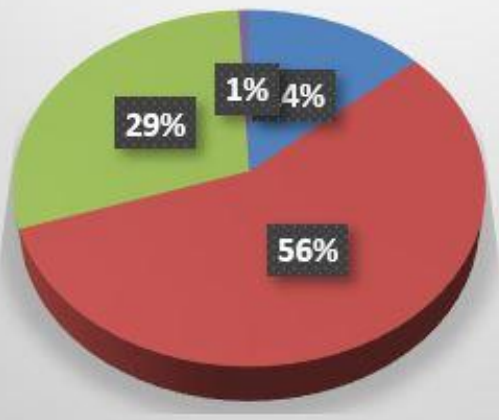

Muy difícil

Dificil

nencillo

muy sencillo

Fig. 5. Gráfico de resultados relativo a la percepción de dificultad del uso de SIG $(n=126)$.

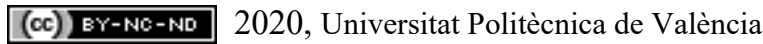

Congreso In-Red (2020) 
Aplicación y evaluación de metodologías activas de enseñanza-aprendizaje que faciliten la adquisición de competencias relacionadas con los Sistemas de Información Geográfica en los programas de Grado y Máster de la Universidad de La Rioja

En la pregunta $\mathrm{n}^{\circ} 5$, relacionada con la presencia que la enseñanza de los SIG tiene en su plan de estudios, una gran mayoría de los estudiantes, casi un $85 \%$, consideraron que la misma es adecuada. Este resultado llama la atención ya que un porcentaje inferior es el que contestó afirmativamente en la pregunta $\mathrm{n}^{\mathrm{o}} 1$ sobre el conocimiento de lo que es un SIG (Fig. 6).

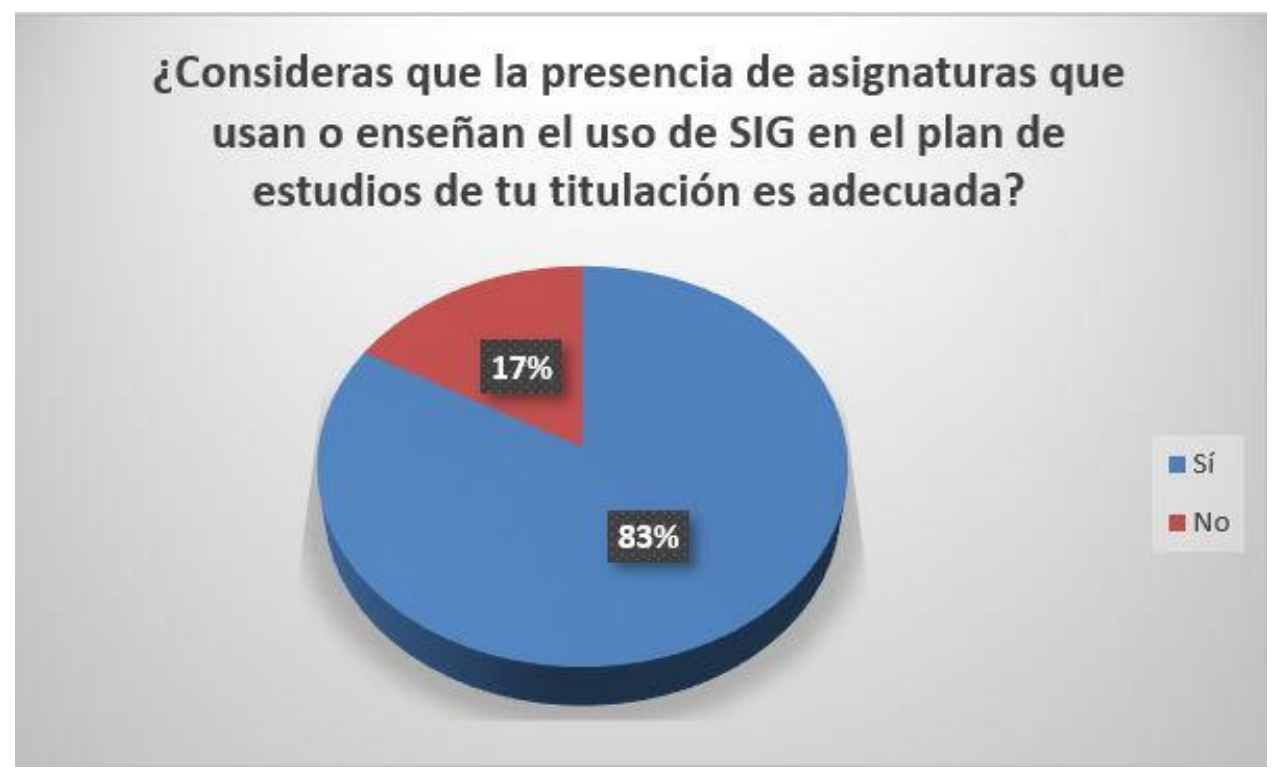

Fig. 6. Gráfico de resultados relativo a la idoneidad de la presencia de SIG en los planes de estudios (n=126).

En la siguiente pregunta, la $6^{\mathrm{a}}$, tratamos de averiguar qué conceptos del ámbito de los SIG eran familiares a los estudiantes. Es llamativo cómo casi todos los estudiantes indicaron conocer algunas herramientas propias de Google, pero luego desconocían en un alto porcentaje algunos de los tipos de ficheros básicos para usar en un SIG (un 91,3\% en el caso de los ficheros Shapefile, un 94,6\% en el caso de los ficheros KML). También resulta sorprendente que solo un $22,2 \%$ ó un $28,6 \%$ declaren conocer lo que es una capa ráster o vectorial (de nuevo, conceptos fundamentales para poder usar un SIG con solvencia). Es importante recordar que, en la pregunta 1, un 60\% de los estudiantes habían respondido saber lo que era un SIG (Fig. 7). 


\section{INDICA SI CONOCES LOS SIGUIENTES CONCEPTOS Y TECNOLOGÍAS}

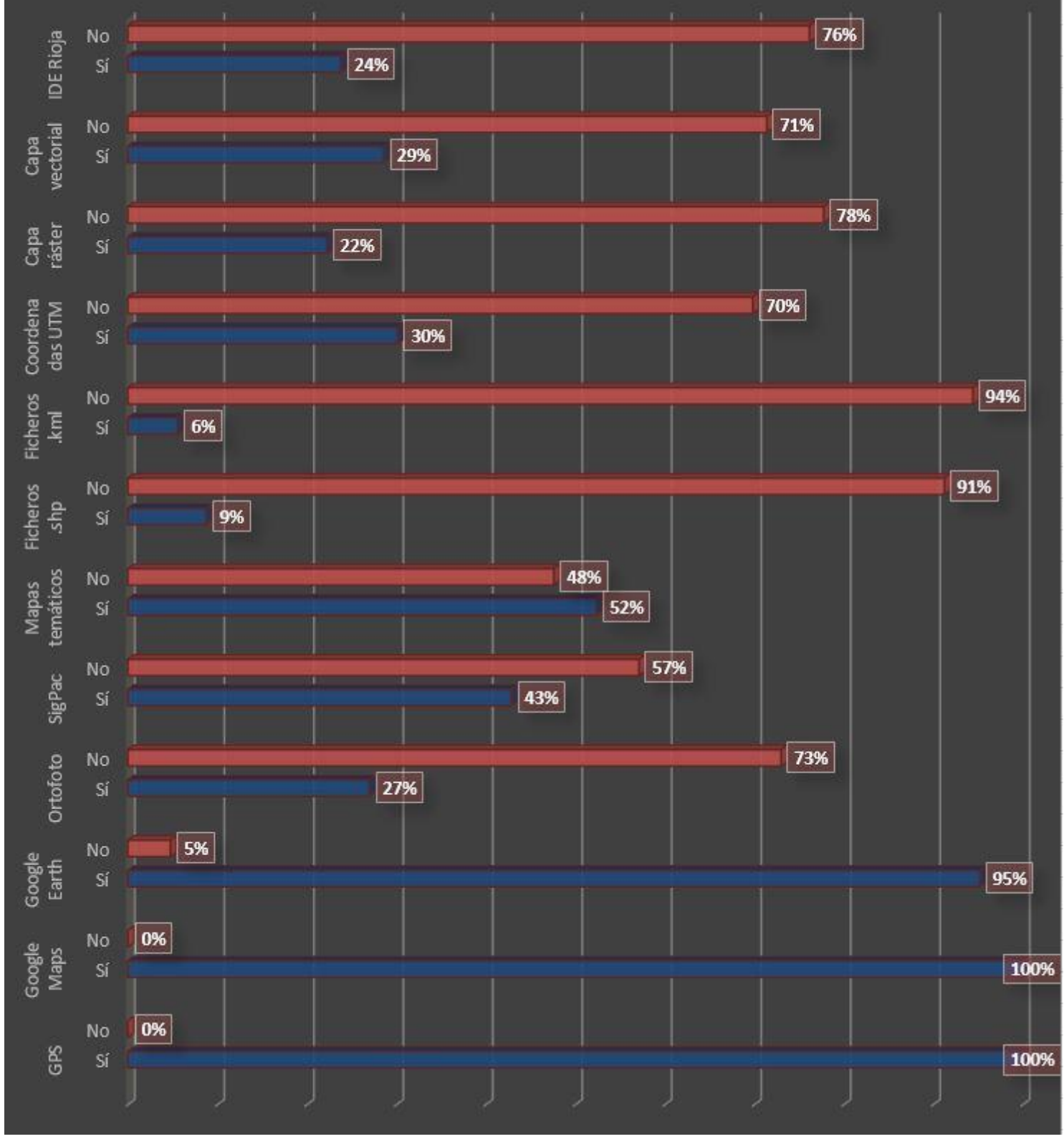

Fig. 7. Resultados de la pregunta acerca de conceptos relacionados con los SIG que les eran familiares ( $n=126)$.

Finalmente, para evaluar también el conocimiento previo de los estudiantes sobre los SIG, se les preguntó acerca de qué aplicaciones de uso cotidiano consideraban que hacen uso de información geográfica. Las respuestas obtenidas refuerzan nuestra percepción de que, aunque un amplio porcentaje de ellos afirmaba saber qué era un SIG, tienen ciertas ideas confusas sobre los mismos. Así lo atestigua el hecho de que un número elevado de los estudiantes identificaron aplicaciones como el correo electrónico (un 64\%) o aplicaciones de música online (p.ej., Shazam, un 38\%) como aplicaciones que hacen uso de información geográfica. Sin embargo, aplicaciones de "tracking" de rutas o recorridos de senderismo (p.ej., Strava, un $49 \%$ ) fueron identificadas por los estudiantes como aplicaciones que no hacen uso de información geográfica (Fig. 8). 
Aplicación y evaluación de metodologías activas de enseñanza-aprendizaje que faciliten la adquisición de competencias relacionadas con los Sistemas de Información Geográfica en los programas de Grado y Máster de la Universidad de La Rioja

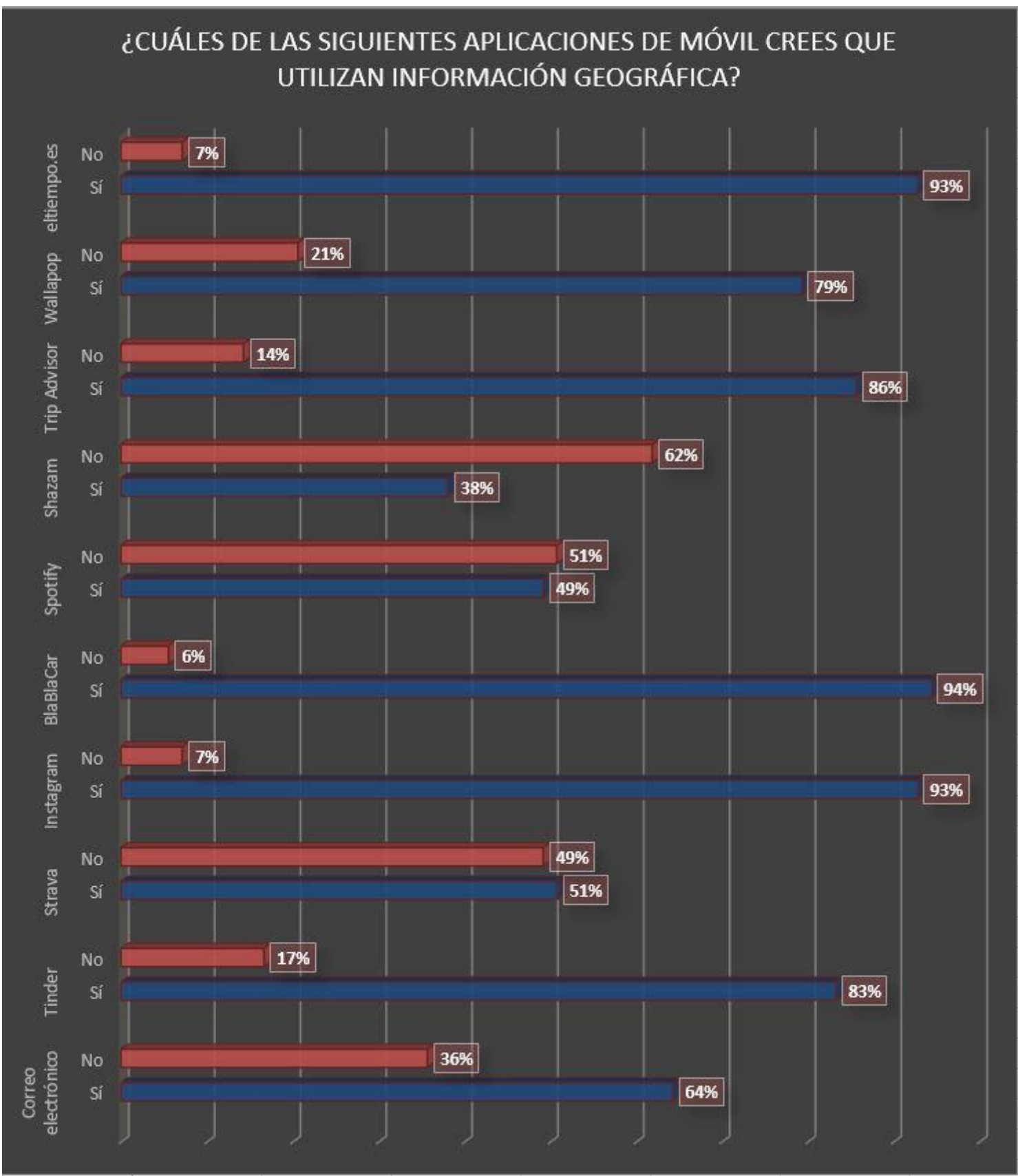

Fig. 8. Resultados de la pregunta relacionada con las aplicaciones de móviles que hacen uso de datos geográficos (n=126).

Los resultados mostrados son el punto de partida del desarrollo del proyecto de innovación docente descrito en la metodología. Como se ha mencionado, de forma paralela a la aplicación del material didáctico (Andrades et al. 2020) se elaborará un cuestionario de autoevaluación o rúbrica que permita valorar la eficacia de la metodología aplicada. El cuestionario va a incluir indicadores de progreso medibles, que pueden ser confrontados con el cuestionario inicial, cuyos resultados han sido discutidos en esta comunicación, para poder evaluar la eficacia de la metodología aplicada y diseñada para el aprendizaje de los SIG en diferentes Grados y Másteres de titulaciones de disciplinas muy diversas, del ámbito de la Ciencia, Tecnología, Humanidades, etc. 


\section{Conclusiones}

El análisis de las respuestas obtenidas a partir del cuestionario de evaluación realizado nos ha permitido identificar el nivel de conocimiento en el tratamiento de datos geográficos y de SIG de los estudiantes, con independencia de la disciplina. Se ha implementado la metodología y el material didáctico publicado. La estructura de este material responde precisamente al análisis previo a su propia redacción y por ello al nivel de uso y demanda de los SIG y datos geográficos de los universitarios. Este trabajo ha permitido mejorar los métodos de enseñanza-aprendizaje llevados a cabo en la Universidad de La Rioja desde una perspectiva multidisciplinar y a partir de formas de actuación que conlleven innovación y coordinación entre profesores de distintas áreas de estudio. Este hecho aporta un valor añadido al trabajar de manera cooperativa distintas asignaturas, aunque con metodologías comunes. Este hecho que fomenta el intercambio de ideas, así como la reflexión crítica entre docentes involucrados en el progreso educativo universitario.

\section{Referencias}

ANDRADES, M. S., ARANSAY, J. M., DIAGO, M. P., LlORENTE, J. A., SAENZ-DE-CABEZÓN, E., TARDÁGUILA, M. J. (2018). "Análisis del uso de datos geográficos y sistemas de información geográfica en las enseñanzas de grado y master de una universidad" en Actas de las XXIV Jornadas sobre Enseñanza Universitaria de la Informática-. JENUI 2018, $\quad$ Vol. $\quad 3, \quad$ pp. $\quad 367-370 . \quad<$ http://www.aenui.net/ojs/index.php?journal=actas_jenui\&page=article\&op=view\&path\%5B $\% 5 \mathrm{D}=428>[\mathrm{Consulta:} 12$ de marzo de 2019]

ANDRADES RODRÍGUEZ, M., ARANSAY AZOFRA, J. M., DIAGO SANTAMARÍA, M. P., LANA-RENAULT MONREAL, N. S., LLORENTE ADÁN, J. A., RUIZ FLAÑO, P. y SÁENZ DE CABEZÓN IRIGARAY, E. (2020). Enseñanza de Sistemas de Información Geográfica (SIG) en estudios de grado y posgrado en la Universidad de La Rioja. Principios teóricos y ejercicios prácticos. La Rioja: Universidad de La Rioja. ISBN 978-84-09-17400-3.

CABERO ALMENARA, J. (2005). "Las TIC y las universidades: retos, posibilidades y preocupaciones". Revista de la educación superior, 34 (135), pp. 77-100.

GUITERT, M., GUERRERO, A. E., ORNELlAS, A., ROMEU, T. y ROMERO, M. (2008). Implementación de la competencia transversal «Uso y aplicación de las TIC en el ámbito académico y profesional» en el contexto universitario de la UOC. Revista Latinoamericana de Tecnología Educativa, vol. 7 (2), p. 81-89.

LlORENTE, J. A., ARANSAY, J. M., SÁENZ DE CABEZÓN, E., DIAGO, M. P., LANA-REANAULT, N., RUIZ, P. y ANDRÁDES, M. S. (2019). "Uso de Software y datos geográficos en trabajos fin de estudio (TFG y TFM) y Tesis Doctorales en la Universidad de La Rioja (1992-2018)" en IN-RED 2019: V Congreso de Innovación Educativa y Docencia en Red. Universitat Politécnica de Valencia. Disponible en: <http://ocs.editorial.upv.es/index.php/INRED/INRED2019/paper/viewFile/10544/4683> p. $241-251$. [Consultado: 12 de marzo de 2020] 